\title{
Anabases
}

ANABASES Traditions et réceptions de l'Antiquité

18 | 2013

Varia

\section{L'Antiquité gréco-romaine au service de la criminologie. Autour du livre de Ch. Caupenne : Être négociateur au RAID}

Marielle de Béchillon

\section{(2) OpenEdition}

Journals

Édition électronique

URL : http://journals.openedition.org/anabases/4376

DOI : $10.4000 /$ anabases. 4376

ISSN : 2256-9421

Éditeur

E.R.A.S.M.E.

Édition imprimée

Date de publication : 1 octobre 2013

Pagination : 241-246

ISSN : 1774-4296

Référence électronique

Marielle de Béchillon, « L'Antiquité gréco-romaine au service de la criminologie. Autour du livre de Ch. Caupenne : Être négociateur au RAID ", Anabases [En ligne], 18 | 2013, mis en ligne le 01 novembre 2016 consulté le 20 octobre 2019. URL : http://journals.openedition.org/anabases/4376 ; DOI : 10.4000/ anabases. 4376

Ce document a été généré automatiquement le 20 octobre 2019

(c) Anabases 


\title{
L’Antiquité gréco-romaine au service de la criminologie. Autour du livre de Ch. Caupenne : Etre négociateur au RAID
}

\author{
Marielle de Béchillon
}

1 Dans quel ouvrage trouve-t-on : comme emblème un vase antique représentant CEdipe assis face à la Sphinge, une référence à Érostrate qui brûla le jour de la naissance d'Alexandre le Grand le temple d'Artémis à Éphèse, une base informatique nationale nommée OEDIP, des références à la rhétorique antique au travers de Gorgias, Démosthène, Quintilien, Cicéron, ou encore à la philosophie et à la dialectique avec Platon, Socrate, Aristote et Zénon d'élée, des citations d'Horace et de Sénèque, une évocation de l'aristie antique accompagnée d'extraits commentés de l'Iliade mettant en scène Achille au combat? Dans le livre de Christophe Caupenne, Être négociateur au $R A I D^{1}$. L'auteur nous invite à ce constat : l'Antiquité gréco-romaine est, une fois encore, au service de la criminologie ${ }^{2}$.

2 Les écrits de Ch. Caupenne constituent un vade mecum de ce qui est devenu, au fil des ans, une discipline riche et aboutie, la négociation : celle que pratique le RAID dans la France d'aujourd'hui pour dénouer les situations de crises ${ }^{3}$. Ch. Caupenne nous donne un aperçu, fort bien documenté, de sa raison d'être, de ses techniques, de ses buts et de ses difficultés. Il offre une synthèse édifiante de sa pratique et des acquis théoriques qui en sont le soubassement.

3 Parmi les compétences requises pour l'exercice de ce métier hautement pluridisciplinaire, celles qu'enseigne la criminologie sont essentielles. Posséder une connaissance fine et opérationnelle des délinquants, saisir leurs profils, comprendre les facteurs et émotions qui déclenchent les passages à l'acte s'avèrent indispensables. Une appréciation subtile des victimes et de leur comportement se révèle tout aussi nécessaire. Enfin - et c'est l'un des apports de Ch. Caupenne - il faut être bien conscient que la négociation est une technique à part entière et qu'elle ne s'improvise pas. Or 
tout cela - les criminologues du XIX ${ }^{e}$ siècle le savaient déjà - se nourrit au mieux de la référence à l'Antiquitét.

4 L'auteur nous invite à nous poser deux questions : pourquoi et comment l'Antiquité gréco-romaine est-elle ainsi au service de la criminologie?

\section{Pourquoi l'Antiquité gréco-romaine est-elle au service de la criminologie?}

5 L'explication est présente dès les premières pages. Ch. Caupenne répète à l'envi qu'il côtoie des tragédies humaines ${ }^{5}$, avec leur lot d'agresseurs, de victimes et d'infractions. Mais l'outil dont il use pour résoudre la crise est plus inattendu: c'est la parole. "Pourquoi serions-nous condamnés, écrit-il, à n'avoir que la violence comme solution à la confrontation? C'est face à cette évidence que vient se positionner la négociation moderne. Elle est devenue, au fil du temps, une voie royale vers laquelle tendent tous ceux qui sont confrontés à des oppositions. La raison est simple, elle porte l'espoir d'une résolution pacifique des situations de conflits ${ }^{6}$.»

D'un côté, les criminels et leurs victimes ; de l'autre, l'usage de la parole au travers de la négociation. Or Ch. Caupenne, dans le sillage des Ferri, Corre, Maxwell et Gardikas, se tourne vers l'Antiquité gréco-romaine pour puiser des connaissances et un savoirfaire ${ }^{7}$. Il constate que les Grecs et les Romains s'étaient déjà posé les questions auxquelles il se trouve confronté au quotidien. Les interrogations qui traversaient les Tragiques grecs, Platon, Aristote, Sénèque, Horace, sont celles qui le taraudent : qu'estce qu'un criminel ? Existe-t-il des différences entre les délinquants? De quelle nature sont ces différences? Pourquoi et comment un individu passe-t-il à l'acte ? Quels sont les facteurs qui le déterminent à agir? Qu'est-ce qu'une victime? Ont-elles un rôle à jouer dans le passage à l'acte des agresseurs ? Comme ses lointains prédécesseurs ${ }^{8}, \mathrm{Ch}$. Caupenne constate la permanence d'un questionnement, la richesse et la pertinence des réflexions véhiculées tout au long de l'Antiquité. Observons maintenant comment il utilise cet apport.

\section{Comment l'Antiquité gréco-romaine vient-elle au service de la criminologie?}

7 Ch. Caupenne y puise des symboles, des modèles et un outil.

8 Le symbole, c'est d'abord l'emblème du groupe figuré par la « représentation d'un vase antique présentant Edipe assis face au Sphinx. Il y a dans cette métaphore antique toute la justification du travail des négociateurs : «Libérer la cité du péril qui la soumet (et, par extension, rendre sa liberté aux otages) ${ }^{9}$."

9 Les modèles: Ch. Caupenne s'efforce de cerner et de catégoriser les actes, les agresseurs, les victimes et les modalités du passage à l'acte. Pour ce faire, il se tourne vers l'Antiquité gréco-romaine. Écoutons-le: «L'attaque aveugle de la population par un individu exalté, prêt à massacrer des dizaines de passants innocents, ou ses collègues de bureau. Ce genre d'acte, d'origine "éro-stratique" s'est considérablement multiplié ces dernières années ${ }^{10}$.» Il s'explique: «Aujourd'hui encore, dans notre criminologie moderne française, on parle d'acte érostratique pour qualifier un passage à l'acte animé 
par la volonté de réaliser un acte sordide, qui marquera les esprits et fera entrer son auteur dans le panthéon des criminels. L'expert psychiatre D. Zagury parle à ce propos d'un "éprouvé subjuguant", longtemps fantasmé, qui aboutit à la décision de passage à l'acte extrême. L'homme devient obnubilé par la réalisation de son geste, jusqu'à ce qu'il devienne une mission "quasi divine"11. » Plus loin, dans un chapitre consacré aux auteurs et aux victimes. Ch. Caupenne envisage les principales émotions de la crise. Il inscrit cette problématique dans les principes généraux de la criminologie et rappelle qu' " une prise d'otage est un système à part entière où les choses (les gens et les événements) interagissent ». Il prévient qu'il faut chercher à comprendre pourquoi les choses en sont arrivées là et qui est "partie prenante ${ }^{12}$ ». Il prône "une vision "systémique" [seule à permettre] une acuité et une discrimination efficaces ${ }^{13}$ ". Il classe aussi les émotions qui commandent la crise humaine : la peur, la colère, la tristesse et le dégoût ${ }^{14}$. Soucieux d'expliquer la colère, l'auteur se réfère à Horace et à Sénèque : « Le poète latin Horace la nommait "la courte folie", mais c'est le philosophe Sénèque qui en parla le mieux: "Car non moins impuissante à se maîtriser, elle oublie toute décence, méconnait les nœuds les plus saints ; opiniâtre, acharnée à son but, sourde aux conseils et à la raison, elle s'emporte pour de vains motifs, incapable de discerner le juste et le vrai [...]" ; le plus souvent, dira-t-on, l'homme s'irrite non contre les gens qui lui ont fait $\mathrm{du}$ tort, mais qui doivent lui en faire, preuve que la colère ne vient pas uniquement de l'offense. Oui, sans doute le pressentiment du mal irrite, mais c'est que l'intention est déjà une injure et que la méditer, c'est l'avoir commise ${ }^{15}$." Plus loin, concernant le dégoût, il écrit: "Souvenons nous que les émotions sont les arcanes de la crise. Retenons cette maxime de Sénèque : "Les passions sont aussi mauvais instruments que mauvais guides"16."

10 Enfin, pour nommer "l'état physique et psychique "anormal" dans lequel se trouvent certains individus soumis à une rage folle, à la détermination homicide irrépressible, ou à un instinct de survie exceptionnel, qui décuplent leurs capacités naturelles: force physique, résistance à la douleur, ou aux contraintes extérieures, endurance hors norme, précision au tir, sixième sens", Ch. Caupenne reconnaît utiliser "un barbarisme qui lui sert à décrire une situation très précise, proche de l'aristie antique ${ }^{17}$ »: l'état de crise «aristéique». "Ce processus de hargne combattante, de fureur agressive tendant à sublimer les capacités d'un individu fait immédiatement penser à un mécanisme appartenant à la mythologie qui fut décrit à de multiples reprises dans l' Iliade : l'aristie, série d'exploits individuels accomplis par un héros en transe guerrière, qui le fait entrer dans la légende et rend son nom digne d'être chanté. L'invulnérabilité d'Achille, héros légendaire de la guerre de Troie, est le symbole de cet état ${ }^{18}$. » Au cours des pages qui suivent, $\mathrm{Ch}$. Caupenne reprend des passages de l'Iliade pour illustrer ses propos. En voici quelques-uns. D'abord l'auteur retient que le héros ne recule pas, ne se rend jamais. Il préfère plutôt mourir : « Ne parle pas de fuite. Je ne suis pas de race, en effet à combattre en me dérobant ni à me terrer. Ma vaillance est encore assurée $(\mathrm{V}, 106)^{19}$. » Ensuite, l'auteur constate que le héros ne pense plus à se nourrir, lors de sa préparation au combat. Les dieux et les déesses bienveillants lui apportent des «substances divines » afin de le faire tenir : «Et tandis que les autres vont prendre leur repas, [Achille] reste à jeun et sans être nourri » (XIX, 465-466). Enfin, le héros est en transe. Il est porté par une fureur sacrée, qui le fait briller, au sens propre comme au sens figuré dans la bataille et décuple ses capacités, jusqu'à les rendre surnaturelles : « Il grinçait des dents, ses yeux brillaient comme l'éclat du feu, et son cœur se sentait pénétré d'une intolérable douleur. Ainsi donc, dans sa fureur contre les Troyens, il 
s'enfonça dans les présents du Dieu, dans l'armure que lui avait façonnée le labeur d'Héphaestos. Or ces armes étaient pour lui comme des ailes; elles soulevaient le pasteur des guerriers » $(\mathrm{XIX}, 466-467)^{20}$.

11 Un outil : la parole. À deux reprises, Ch. Caupenne invoque l'Antiquité lorsqu'il parle de la négociation. "L'écoute est notre arme ${ }^{21}$ ", dit-il. Reprenant à son compte la formule du préfet Ange Mancini - chef historique du RAID -, il écrit : «La négociation, ce n'est pas préférer les mots aux actes, mais utiliser la parole comme une arme ${ }^{22}$.» Ch. Caupenne inscrit les principes de la négociation dans le droit fil de l'art oratoire, " premier outil de la communication d'influence » selon ses propres termes ${ }^{23}$. C'est bien de rhétorique qu'il s'agit ${ }^{24}$, ici définie comme l'art de l'éloquence et de la persuasion. Ancrant la discipline dans la Grèce antique, notre auteur en retrace brièvement les grandes étapes ${ }^{25}$.

Tout antiquisant se réjouira de retrouver un peu de son objet d'étude sous le "calame " moderne d'un homme d'action. Un dernier point mérite l'attention: quelle est la nature d'une telle réception de l'Antiquité? Nous serions tentés d'envisager deux manières distinctes de la concevoir ici bien qu'elles ne soient pas séparées. Il y a d'abord ce que nous qualifierons de « réception-héritage ». Dans cette optique, ce que l'on retient de l'Antiquité s'inscrit dans un lien culturel qui perdure depuis les temps reculés : les questions sur les criminels, par exemple, illustrent cela très bien. Mais on entrevoit aussi une autre forme de réception, certainement plus présente dans cet ouvrage et que nous pourrions nommer « réception-importation ». L'acte érostratique ou l'acte aristéique procèdent de cet emploi. Il ne s'agit pas de recueillir un héritage, mais plutôt de saisir en partant de notre époque ce qui, dans l'Antiquité, pourrait nous servir à mieux comprendre et appréhender des problématiques actuelles. Il n'est pas question de savoir si ce que l'on prend avait un sens parfaitement connaissable dans le contexte de l'époque et si on ne le dénature pas en l'utilisant de nos jours, mais fondamentalement de faciliter l'analyse ou la connaissance d'un phénomène, d'un état ou d'une situation. Cette forme de réception se retrouve dans bien d'autres disciplines. Il en va ainsi, par exemple, lorsque l'on transpose des concepts d'un domaine à un autre en utilisant leur puissance d'évocation. Toutes choses égales par ailleurs, cet emploi fonctionne un peu à la manière d'une métaphore. C'est ce que fait Boris Cyrulnik, par exemple, lorsqu'il retient des sciences physiques le concept de résilience pour décrire les capacités de résistance de victimes de traumatismes ${ }^{26}$. C'est ce qu'avait probablement fait Charles Darwin lorsqu'il avait emprunté le mot de « sélection » au vocabulaire de l'horticulture pour faire saisir ce qu'il avait à l'esprit... Quelle science peut se passer d'user des meilleurs moyens de (se) faire comprendre, et donc des vertus d'une importation judicieuse? Soit dit presque par parenthèse, c'est une bien grande réjouissance de constater que, peut-être même à notre corps défendant, l'Antiquité continue - jusque dans les vestiges d'elle qui demeurent dans la culture de tous - de donner de quoi nous éclairer là où nous ne voyons pas clair.

Édifiant dans sa capacité à convoquer l'Antiquité gréco-romaine, l'ouvrage de Ch. Caupenne nous invite donc aussi à méditer sur la manière dont on la reçoit de nos jours, comme sur l'utilité que l'on peut y trouver même là où on ne l'attend pas. 


\section{NOTES}

1. Ch. CAUPENNE, ÊTRE NÉGOCIATEUR AU RAID, PARIS, LE CHERCHE MIDI, 2011.

2. Par convention, nous reprenons ici une définition consensuelle de la criminologie: c'est «l'étude scientifique du phénomène criminel » ou « la science du crime ». R. GASSIN, CRIMINOLOGIE, DALLOZ, $6^{E}$ ÉD. 2007, P. 3. ELLE NE PEUT ÊTRE CONFONDUE AVEC LE DROIT STRICTO SENSU. MAIS ELLE CONTRIBUE, DE PLUS EN PLUS, À PARFAIRE LA RÉPONSE JURIDIQUE AU PHÉNOMÈNE CRIMINEL. ELLE DEVIENT AINSI UNE DISCIPLINE UTILE ET SOUVENT CONVOQUÉE POUR L'ÉLABORATION DU DROIT ET PLUS PARTICULIÈREMENT DU DROIT PÉNAL.

3. Le RAID, DONT L'ACRONYME SIGNIFIE « RECHERCHE, ASSISTANCE, INTERVENTION ET DISSUASION » EST UNE UNITÉ DE LA POLICE NATIONALE FRANÇAISE CRÉÉE EN 1985 (P. 9). LE MÉTIER DE « NÉGOCIATEUR DE SITUATION DE CRISE » A ÉTÉ RECONNU PAR UN ARRÊTÉ MINISTÉRIEL DU 14 JUIN 2006 (P. 36). CH. CAUPENNE EST RESTÉ PENDANT 10 ANS LE CHEF DES NÉGOCIATEURS DU RAID. IL PRÉCISE AINSI SA MISSION : «CETTE FORME DE NÉGOCIATION N’A RIEN À VOIR AVEC LES AUTRES FORMES DE NÉGOCIATION. ELLE S'ATTACHE À DES CRISES DITES EXTRÊMES, C'EST-À-DIRE DONT LES ENJEUX CRIMINOLOGIQUES SONT LA VIE OU LA MORT D'ÊTRES HUMAINS » (P. 30).

4. Entendons-nous bien: Ch. Caupenne mentionne bien d'autres disciplines dans son ouvrage. Nous ne retenons, dans le cadre de cet atelier, que ce qui concerne l'Antiquité gréco-romaine.

5. Ch. Caupenne, Être négociateur au RAID, p. 14, 15, 16, cf. la répétition du mot Tragédie.

6. Ibid., p. 27.

7. E. FERRI, LES CRIMINELS DANS L'ART ET LA LITTÉRATURE, ALCAN, 1897. A. CORRE, PLATON CRIMINALISTE, ARCHIVES D'ANTHROPOLOGIE CRIMINELLE, 1908, P. 19 ET J. MAXWELL, LE CONCEPT SOCIAL DE CRIME ET SON ÉVOLUTION, ALCAN, 1914. C. GARDIKAS, QUELQUES CONSIDÉRATIONS SUR LE DROIT PÉNAL ATTIQUE ET LES @UVRES DES TRAGIQUES GRECS, EXTRAIT DE LA REVUE DE DROIT PÉNAL ET DE CRIMINOLOGIE, 1923, P. 791.

8. Cf. en ce sens E. FERRI : «LA TRAGÉDIE GRECQUE EXPRIME PARFOIS AVEC UNE PROFONDE INTUITION DE LA RÉAlité, L'IDÉE DE LA FATALITÉ QUI PÈSE SUR LA CRÉATION CRIMINELLE », P. 33. AILLEURS, L'AUTEUR AFFiRME: «AINSI DÈS SES PREMIERS CHEFS-D'œUVRE, L'ART OCCIDENTAL A SU ESQUISSER LES TROIS PRINCIPAUX TYPES DE CRIMINELS : LE CRIMINEL-NÉ, LE CRIMINEL-FOU (MÉDÉE) ET LE CRIMINEL PAR PASSION (ORESTE ET PHÈDRE) », P. 34 ET 35.

9. P. 39. Le logo a été créé en 2000 par le psychologue du RAID, CHRISTOPHE BAROCHE.

10. P. 31.

11. P. 32. Ch. Caupenne donne quelques exemples récents d'actes ainsi diagnostiqués: celui de Tim Kretschmer, 19 ans, qui perpétra en 2009 un massacre dans une école allemande. Et, plus connu encore, l'acte de l'homme que l'on surnomma Human Bomb, E. Schmitt, qui avait retenu et menacé de mort 21 enfants d'une école maternelle à Neuilly-sur-Seine du 13 au 15 mai 1993.

12. P. 233.

13. P. 233.

14. P. 234 à 241.

15. P. 235 à 236.

16. P. 238.

17. P. 250.

18. P. 261.

19. P. 262.

20. P. 262.

21. C'est d'ailleurs la devise qui accompagne l'emblème du groupe : le vase antique représentant ๔dipe et la Sphinge.

22. P. 39.

23. P. 339.

24. P. 44. 
25. P. 43. Il retrace dans l'annexe V l'histoire de la rhétorique en nommant les Grecs et les Romains qui s'illustrèrent dans cette discipline. Il consacre des développements plus nourris aux travaux de Quintilien, rhéteur et pédagogue romain du $\mathrm{I}^{\mathrm{ER}}$ SIÈCLE, NOTAMMENT EN RAPPELANT LES CINQ PARTIES DE L'ART ORATOIRE: INVENTIO, DISPOSITIO, ELOCUTIO, ACTIO, MEMORIA. CES PRÉCISIONS PRENNENT CERTAINEMENT TOUT LEUR SENS QUAND ON SAIT À QUEL POINT LES TECHNIQUES DE NÉGOCIATIONS ACTUELLEMENT EN VIGUEUR SONT MISES AU POINT ET CLASSIFIÉES AVEC UNE RIGUEUR QUI N’EST PAS SANS RAPPELER CELLE DE QUINTILIEN. EN CE SENS CF. P. 36.

26. B. CYRULNiK, LES VILAINS PETITS CANARDS, PARIS, OdILE JACOB, 2004. DANS LE MÊME ÉTAT D’ESPRIT CF. ÉGALEMENT I. STENGERS (DIR.), D’UNE SCIENCE À L'AUTRE. DES CONCEPTS NOMADES, PARIS, SEUIL, 1987.

\section{AUTEUR}

\section{MARIELLE DE BÉCHILLON}

Maître de Conférences de Droit privé

Université de Pau

PLH-ERASME (EA 4601)

marielle.debechillon@neuf.fr 